\title{
Cinema Image-based In Situ Analysis and Visualization of MPAS-Ocean Simulations
}

\author{
Patrick O'Leary ${ }^{\mathrm{a}, *}$, James Ahrens ${ }^{\mathrm{b}}$, Sébastien Jourdain ${ }^{\mathrm{a}}$, Scott Wittenburga ${ }^{\mathrm{a}}$, \\ David H. Rogers ${ }^{\mathrm{b}}$, Mark Petersen ${ }^{\mathrm{b}}$ \\ ${ }^{a}$ Kitware, Inc. 1800 Old Pecos Trail, Suite G, Santa Fe, NM 87505 \\ ${ }^{b}$ Los Alamos National Laboratory, Los Alamos, NM 87545
}

\begin{abstract}
Due to power and I/O constraints associated with extreme scale scientific simulations, in situ analysis and visualization will become a critical component to scientific exploration and discovery. Current analysis and visualization options at extreme scale are presented in opposition: write files to disk for interactive, exploratory analysis, or perform in situ analysis to save data products about phenomena that a scientists knows about in advance. In this paper, we demonstrate extreme scale visualization of MPAS-Ocean simulations leveraging a third option based on Cinema, which is a novel framework for highly interactive, image-based in situ analysis and visualization that promotes exploration.
\end{abstract}

Keywords: High performance computing, In situ, Image-based, Analysis and Visualization

\section{Introduction}

Extreme scale ( $\geq 10^{15}$ FLOPS) parallel computing systems require scientists to change the manner in which they analyze and visualize simulation results. Until recently, data, at significantly smaller scales, have been primarily stored and, typically, moved to other machines for post-processing. Transitioning to next-generation computing systems means that these standard post-processing techniques will not be able to effectively scale due to availability of storage and the relatively slow increase in both storage and network bandwidth.

In situ techniques that analyze and visualize simulation data in simulation memory are at least one promising path forward. In general, in situ approaches operate on a predefined set of analyses and visualizations. In order for in situ analysis and visualization to meet the needs of scientists at extreme scale, it should: (1) preserve important elements of the simulations, (2) significantly reduce the data needed to preserve these elements, and (3) offer as much flexibility as possible for post-processing exploration.

\footnotetext{
* Corresponding author

Email address: patrick.oleary@kitware.com (Patrick O'Leary)
}

Preprint submitted to Journal of Parallel Computing Systems \& ApplicationsSeptember 15, 2015 
In this paper, describing the visualization of a large-scale Model for Prediction Across Scales (MPAS) Ocean simulation, we leveraged the Cinema framework $[1,2]$. Cinema is a novel framework for highly interactive, image-based in situ analysis and visualization that promotes interactive exploration. This interactive exploration - so important to scientific discovery - is supported intuitively. Cinema effectively preserves this capability by allowing in situ data products to be combined in much the same way they could be in a postprocessing tool like ParaView.

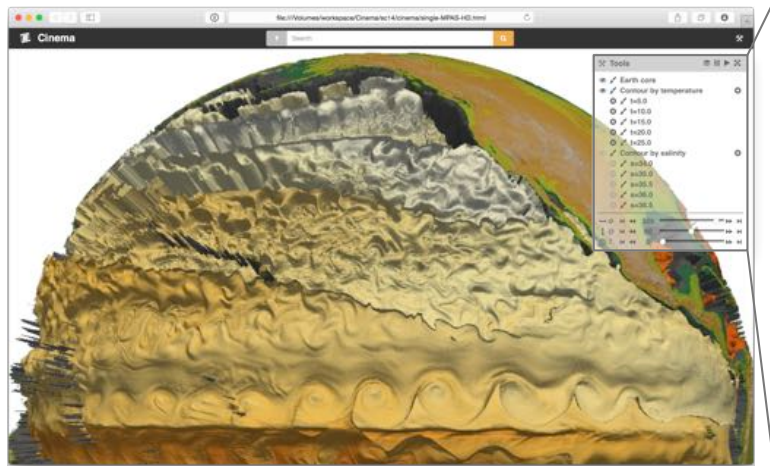

(a)

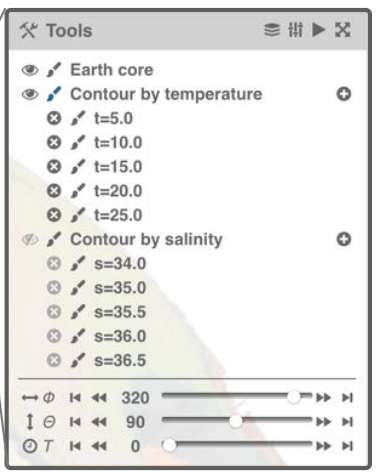

(b)

Figure 1: (a) MPAS-Ocean simulation indicating isosurfaces that represent the locations of water masses within the ocean of temperature colored by salinity. (b) The user interface of the visualization pipeline for analysis and visualization objects (Earth core, temperature contours, and salinity contours) compositing.

Cinema imagery is on the order of $10^{6}$ in size, whereas extreme scale simulation data is on the order of $\geq 10^{15}$ in size. This provides the scientist with $10^{9}$, or greater, images to facilitate capture, curation, and exploration of important elements (features) in the simulation. Cinema is an in situ exploration tool used in a traditional in situ operational mode, but it allows us to sample the analysis and visualization parameter space, such as camera positions, analysis and visualization objects, time, etc., to produce a set of images stored in a dataintensive database. A positive attribute of Cinema is derived from the fact that the analyses and visualizations are created from full-resolution data with high accuracy.

The visualization shown in Figure 1(a) (as well as a video available at https://vimeo.com/127075375) is a run of MPAS-Ocean. MPAS [3] is a new software framework for the rapid development of climate model components on unstructured grids. MPAS-Ocean provides large-scale parallel scalability, expected in a next-generation code, and support for variable resolution meshes. MPAS is developed cooperatively by Los Alamos National Laboratory and the National Center for Atmospheric Research for the purpose of decadal to centurylong climate change research, as well as short-term weather forecasting.

The high-resolution global ocean simulation with realistic topography, uses 1.8 million horizontal $15 \mathrm{~km}$-sized grid cells and 40 vertical levels. The isosur- 
faces of temperature and salinity indicate the locations of water masses within the ocean. Water masses, with names like North Atlantic Deep Water and Antarctic Bottom Water, occur within specific ranges of temperature and salinity. These visualizations allow oceanographers to view the pathways and extents of these water masses, and compare them to observed climatology. Meandering ocean jets and eddies are visible as perturbations to these analysis and visualization objects, and the ocean currents and eddy activity compare well with observations.

These simulations are typically run on 6000 processors, and achieve two simulated years per wall clock day. At this resolution, file output sizes present difficulties for traditional post-processing analysis and visualization workflows.

\section{Related Work}

Cinema makes a number of contributions, while building on a vast amount of previously disseminated results in interactive exploration databases, creating new visualizations and metadata and content searching.

Interactive Exploration Database. A large image collection is produced from a structured sampling of time, analysis and visualization objects and camera positions. One option for the management of this large image collection is to compress them into movies $[4,5]$. Cinema extends this approach by support compositing of images to create new visualizations as well as metadata and content searching. Tikhonova et al. $[6,7,8]$ represent data as a collection of proxy images. Combining the two approaches would support additional data reduction and exploration possibilities.

Creation of New Visualizations. Cinema supports combining images using depth ordering information, while Tikhonova et al. [6] creates new visualizations using interpolation. Cinema's idea for compositing visualization results evolved from the long history of parallel compositing techniques that enable scalable interactive visualization [9].

Metadata and Content Searching. Recent work by Subrahmaniam [10] identifies issues and future research directions for multimedia databases. Cinema creates imagery in situ saving into its database: camera positions, time, details about the analysis and visualization objects, and statistics about the data. Directly storing analysis results and how they are created is important and encompasses our connection to provenance systems [11].

Image content queries support querying about the visual weight of the objects in the generated visualization. There are many approaches to calculating the statistics of the 2D projection of a set of $3 \mathrm{D}$ objects [12]. Related to our approach, Jun Tao et al. [13] computes a collection of streamline images, and applies an image quality metric to select an optimal viewpoint. Our approach extends this work by virtue of being in situ and our ability to change our evaluation metric dynamically with a scientist-generated query. 


\section{Discussion}

Interactive exploratory post-processing analysis and visualization will still be essential for scientific discovery at extreme scale. First, debugging new simulators requires this support. Second, collaboration for colleagues without equal computing resources need this support. Finally, transitioning existing simulators demands this support for a path from terascale/petascale $\left(10^{12} / 10^{15}\right.$ FLOPS) to extreme scale.

\subsection{From Simulation Data To Image Database}

Cinema, currently, leverages ParaView [14] to define a desired set of analysis and visualization objects based on a test data set. At this point, scientists can make sophisticated prioritized decisions for the production of Cinema output. They can define how to sample: time, analysis and visualization object parameter space, camera space, and image resolution. The results of these choices are reported by cost metrics associated with the number of images, the image size, the imagery size, and additional computational time.

As simulations progress towards extreme scale, scientists will operate in a constrained storage and bandwidth environment. Hence, sampling of simulation results will require prioritization to fit within a storage/bandwidth budget. Cinema was designed to operate within these constraints, and can assist scientists in making appropriate decisions.

For the in situ analysis and visualization, we use Catalyst, an open-source in situ data analysis and visualization optimized C++ library, which includes an API for C, FORTRAN, Python and C++. Catalyst is designed to be tightly coupled by directly embedding it into parallel simulation codes.

As the Catalyst enabled simulation runs, the Cinema results are ingested into a database. By this we mean, that the metadata, image provenance (i.e., a searchable description of how the image was created - the simulation, the input deck, and which objects were created), and the image uniform resource locator (URL). Therefore, there is potentially no need to move the potentially large image data around in bulk.

\subsection{Creation of New Visualizations}

Cinema utilizes real-time compositing to create an experience reminiscent of interactively exploring the raw simulation data. For the Web-based Cinema viewer, compositing is enabled through WebGL using ping-pong shaders. For each object (layer) to be composited (see Figure 2(c)), Cinema loads the data and surface normals into textures. The previous framebuffer object (FBO) is bound to a texture for reading, while the current FBO is bound for writing (rendering). The object is composited by drawing a textured rectangle, and then swapping the current and previous FBOs.

The field data associated with an analysis and visualization object is stored as a 24-bit float in the $\mathrm{r}, \mathrm{g}$, and b components of separate Cinema images. The alpha component stores the depth information. In Figure 2(a), we demonstrate how the scalar value is extracted from the object data texture. Finally, as 

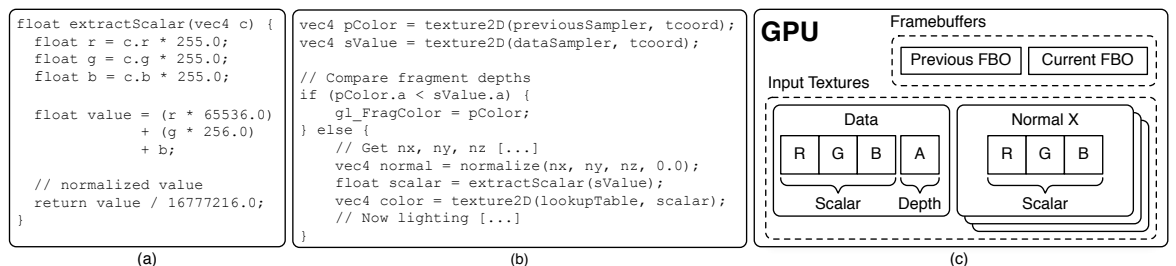

Figure 2: The interactive exploration, enabled by the Cinema graphical user interface (GUI), pulling resulting Cinema images and analysis products from the interactive exploration database.

depicted in the Figure 2(b) fragment shader, for each fragment the depth is tested, and if compositing is required, the normal and scalar components are extracted and used to compute a normal and color for Blinn-Phong lighting calculations.

It's Cinema's user interface that significant enhances the exploration experience seemingly beyond those available in traditional post-processing tools. From a scientist's perspective, computationally intensive analysis and visualization algorithms run in constant time using the precomputed Cinema images and analysis products. The interactive exploration database currently supports three elements and two modes of interaction. The three elements of interaction are time, analysis and visualization objects, and camera. The modes of interaction are: animation; where the interaction is a sequence through time, objects and camera; and selection, where the scientist selects or queries time, objects, and camera.

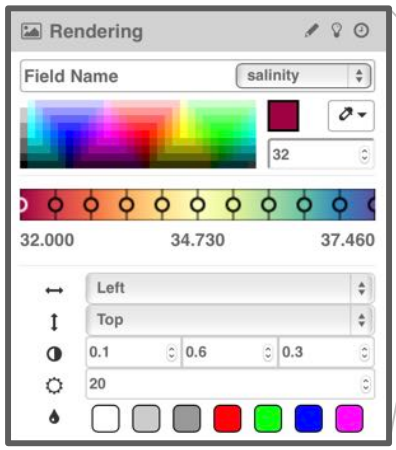

(a)

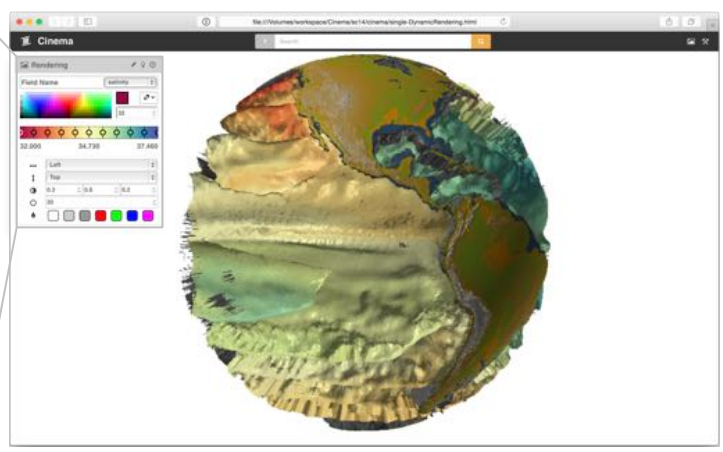

(b)

Figure 3: (a) The dynamic rendering widget offers the capability to edit color lookup tables, set the light direction and color, and adjust material properties. (b) The fully interactive exploration, enabled by the Cinema GUI, pulling resulting Cinema images and analysis products from the interactive exploration database.

We can automatically display multiple objects from visualization space by selecting the associated (time, objects, and camera) Cinema images and analysis products from the database and composing them together. Cinema does not require the scientist to do this manually through a database query. Instead, the scientist uses a graphical user interface (GUI), depicted in Figure 1(b), 
that emulates an application like ParaView to create an experience similar to exploring raw simulation data. In Figure 1(b): the Eyes indicate analysis and visualization objects that can be interactively turned on and off; the Plus and Pencil allow for selection and coloring of specific analysis and visualization objects; and the Time, Phi and Theta controls allow for either selection or animation of these elements.

In addition, the dynamic rendering GUI, shown in Figure 3(a), allows the scientist to further investigate the simulation field data associated with the analysis and visualization objects. It's these types of capabilities that pushes the Cinema experience closer to interactive exploration of raw simulation data.

\subsection{Metadata and Content Querying}

By leveraging the metadata associated with the Cinema images and analysis products in the interactive exploration database, Cinema allows the scientist to execute metadata queries (or browse analysis and visualization objects) to produce a prioritized sequence of matching results, as demonstrated in Figure 4. The metadata, produced by the in situ analysis and visualization process, includes data properties of the simulation data, such as histograms, as well as image properties.

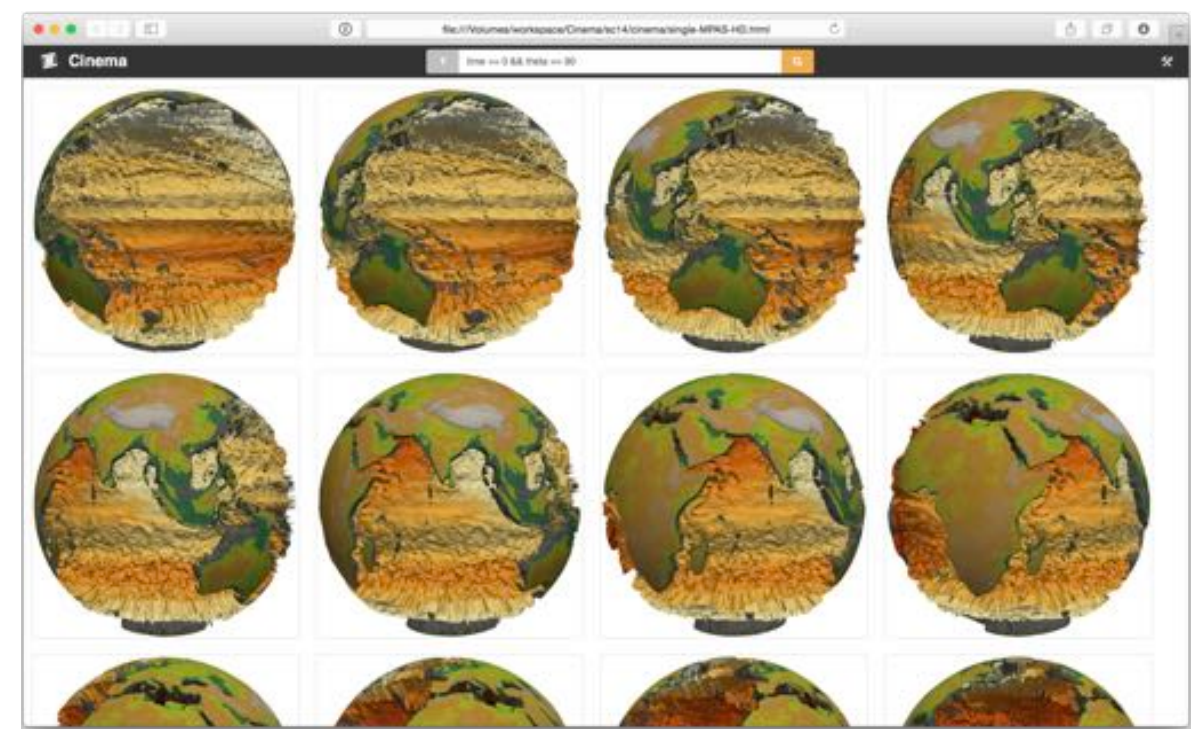

Figure 4: The graphical user interface of the scientist-defined search pipeline for analysis and visualization object compositing.

The compositing infrastructure also makes it possible to perform queries that search on the content of the image in the database. A query could be formulated that matches on the quality of the view of a particular analysis and visualization object value. However, Figure 4 shows a far simpler set of search results associated with a particular compositing pipeline and a query of time $==0$ \&\& theta $==90$. 


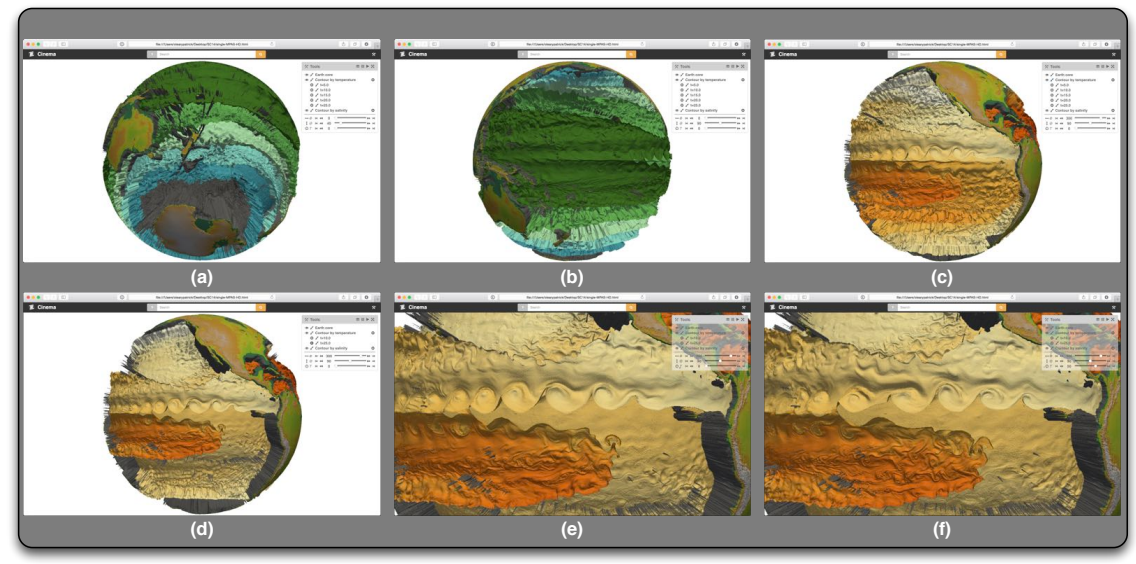

Figure 5: (a), (b) and (c) Demonstrate the Cinema MPAS-Ocean results using rotation about the $\mathrm{x}^{-}, \mathrm{y}$-axis and changing to colored by salinity temperature layers. (d) Depicts editing the composite pipeline by removing three temperature contours. (e) and (f) Show the pan and zoom interaction and the selection of different time steps.

\section{Results}

Figures 5 and 6 demonstrate the interactive capabilities of Cinema for visually exploring the results of the MPAS-Ocean simulation. While data reduction is highly dependent on sampling and the specified visualization pipelines, this simulation claimed an approximately 202 GBs to 16 GBs reduction, but an increase from $101 \mathrm{MBs}$ for a one image per time step traditional in situ workflow.

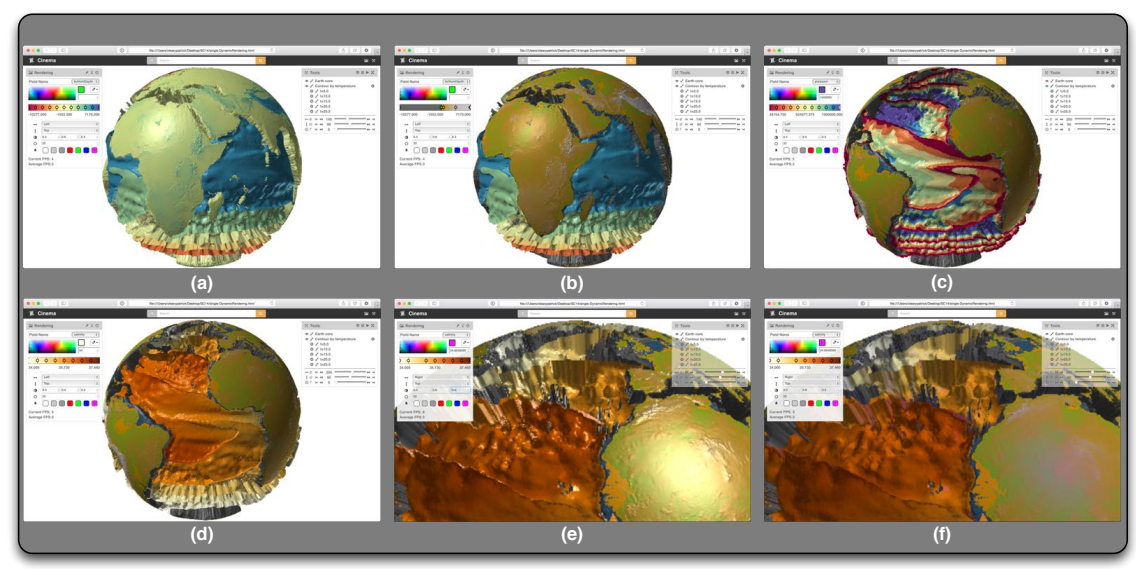

Figure 6: The capabilities added by dynamic deferred rendering. (a) and (b) Show temperature isosurfaces with an Earth core colored with a spectral or an Earth-like colormap. (c) and (d) Demonstrate dynamically coloring by different parameters (pressure and salinity) and colormaps. (e) and (f) Depict increasing the spectral contribution and changing color (blue) of the light. 


\section{Conclusion}

Cinema is a novel framework for image-based extreme scale in situ analysis and visualization. It couples in situ analysis and visualization outputs with an image database query method to enable interactive exploration and data browsing. The compositing pipelines provide an experience that mirrors the interactive exploration of raw simulation data, but with a constant computation time for all analysis and visualization algorithms. As implemented, Cinema meets the overarching goals for in situ analysis and visualization systems. It preserves important elements of simulations, while significantly reducing the data needed to preserve these elements, and offers sophisticated interactive postprocessing exploration.

\section{Acknowledgments}

The authors would like to thank Patricia Fasel ${ }^{1}$, Andrew Bauer ${ }^{2}$, Benjamin Boeckel $^{2}$, Dave DeMarle ${ }^{2}$ and Zach Mullen ${ }^{2}$ for their development work involving either in situ MPAS-Ocean or Cinema. We would also like Francesca Samsel ${ }^{3}$ for the ocean color lookup table.

This work was funded by Dr. Lucy Nowell, ASCR Program, Office of Science and ASC, Department of Energy (DOE). Patrick O'Leary, Sébastien Jourdain and Scott Wittenburg were also funded by a DOE Office of Nuclear Energy Fast Track SBIR award DE-SC0010119.

\section{References}

[1] J. Ahrens, S. Jourdain, P. O'Leary, J. Patchett, D. H. Rogers, M. Petersen, An image-based approach to extreme scale in situ visualization and analysis, in: Proceedings of the International Conference for High Performance Computing, Networking, Storage and Analysis, SC '14, IEEE Press, Piscataway, NJ, USA, 2014, pp. 424-434. doi:10.1109/SC.2014.40.

URL http://dx.doi.org/10.1109/SC.2014.40

[2] J. Ahrens, S. Jourdain, P. O'Leary, J. Patchett, D. H. Rogers, P. Fasel, A. Bauer, M. Petersen, F. Samsel, B. Boeckel, In Situ MPAS-Ocean Image-Based Visualization, Online - The International Conference for High Performance Computing, Networking, Storage and Analysis, Visualization \& Data Analytics Showcase (June 2014).

URL http://sc14.supercomputing.org/sites/all/themes/sc14/ files/archive/sci_vis/sci_vis_pages/svs105.html

\footnotetext{
${ }^{1}$ Los Alamos National Laboratory

${ }^{2}$ Kitware

${ }^{3}$ University of Texas at Austin
} 
[3] T. Ringler, M. Petersen, R. L. Higdon, D. Jacobsen, P. W. Jones, M. Maltrud, A multi-resolution approach to global ocean modeling, Ocean Modelling 69 (2013) 211-232.

[4] J. Chen, I. Yoon, W. Bethel, Interactive, internet delivery of visualization via structured prerendered multiresolution imagery, IEEE Transactions on Visualization and Computer Graphics 14 (2) (2008) 302-312.

[5] A. Kageyama, T. Yamada, An approach to exascale visualization: Interactive viewing of in-situ visualization, Computer Physics Communications 185 (1) (2014) 79-85.

[6] A. Tikhonova, C. D. Correa, K.-L. Ma, Visualization by proxy: A novel framework for deferred interaction with volume data, IEEE Transactions on Visualization and Computer Graphics 16 (6) (2010) 1551-1559.

[7] A. Tikhonova, H. Yu, C. D. Correa, J. H. Chen, K.-L. Ma, A preview and exploratory technique for large-scale scientific simulations, in: Proceedings of the 11th Eurographics Conference on Parallel Graphics and Visualization, EG PGV'11, Eurographics Association, Aire-la-Ville, Switzerland, Switzerland, 2011, pp. 111-120.

[8] A. Tikhonova, C. D. Correa, K.-L. Ma, An exploratory technique for coherent visualization of time-varying volume data, Computer Graphics Forum 29 (3) (2010) 783-792.

[9] K. Moreland, W. Kendall, T. Peterka, J. Huang, An image compositing solution at scale, in: Proceedings of 2011 International Conference for High Performance Computing, Networking, Storage and Analysis, SC '11, ACM, New York, NY, USA, 2011, pp. 25:1-25:10.

[10] V. S. Subrahmanian, S. Jajodia, Multimedia Database Systems: Issues and Research Directions, 1st Edition, Springer Publishing Company, Incorporated, 2012.

[11] J. Freire, D. Koop, F. Chirigati, C. Silva, Reproducibility using vistrails, Implementing Reproducible Computational Research.

[12] N. Elmqvist, P. Tsigas, A taxonomy of 3d occlusion management for visualization, Visualization and Computer Graphics, IEEE Transactions on 14 (5) (2008) 1095-1109.

[13] J. Tao, J. Ma, C. Wang, C.-K. Shene, A unified approach to streamline selection and viewpoint selection for $3 \mathrm{~d}$ flow visualization, IEEE Transactions on Visualization and Computer Graphics 19 (3) (2013) 393-406.

[14] A. H. Squillacote, D. E. DeMarle, J. Ahrens, C. Law, B. Geveci, K. Moreland, B. King, ParaView Guide, 1st Edition, Kitware, Incorporated, 2007. 